\title{
The Study of The Framework For TCM Production Management System
}

\author{
Herui Wang \\ Software Engineering Institute \\ BeiHang University \\ Beijing, China \\ coolherrybh@gmail.com
}

\author{
Chaoying $\mathrm{Wu}$ \\ Software Engineering Institute \\ BeiHang University \\ Beijing, China \\ dianewu@vip.sina.com
}

\begin{abstract}
In combination with a traditional Chinese medicine pharmaceutical factory's current production situation, in view of the various problems such as the poor quality, the low productivity faced in the process of production. This paper solves the poor quality problems such as real-time data acquisition and tracking, storage and data transaction processing problems and through the SCADA system, solves the low productivity problems caused by production scheduling chaos through the MES system, After comparing various data Interaction technology, using the OPC technology to integrate the SCADA system and the MES system. Combining the advantages of SCADA system and MES system to achieve high productivity and quality production of traditional Chinese medicine.
\end{abstract}

Keywords-component; OPC DA ; OPC XML-DA ; SCADA; Real-Time Database; MES

\section{INTRODUCTION}

In recent years, as people's understanding of the limitations of western medicine and the change of the world disease spectrum and the medical model, return to nature, advocating natural medicine is becoming a worldwide trend, which provides unprecedented opportunities for Traditional Chinese Medicine (CTM) development.

As a CTM production enterprise, product quality is the most basic requirement of enterprise production, as we all know, the quality is produced rather than testing, which requires the pharmaceutical production must have advanced process monitoring and quality control method, the current Chinese medicine production process monitoring and quality control methods are too single, mostly is artificial observation and offline detection methods, part of the quality indicators and the key process parameters are not realize the online measurement, which makes the observed process parameters are not accurate, the result of detection and measurement time lag.

To solve these problems, improve the quality of curative, productivity and enterprise competitiveness, improving the level of the workshop production process management is the key.

Currently there are a lot of workshop production management scheme is put forward. The literature [1] puts forward the integration of ERP, MES and PCS, which takes MES as a bridge to ERP and PCS, implementing the enterprise production plan into the workshop production.
The literature [2] designed a MES, which the main line is planning and scheduling and the core is cost control.

These schemes focused on the integration of MES and its upper system, and the design of the MES system module. But they lack of integration of MES and the underlying equipment design. CTM production process will monitor and manage a variety of production equipment. So how to do a good job in the management of the underlying device and the communication of MES with the equipment is very important.

After the analysis of a variety of information and communication technologys, equipment control system, this paper puts forward integrating SCADA system and MES through OPC technology, to achieve the CTM production process automation monitoring and control.

\section{KEY TECHNOLOGIES}

\section{A. Data Interaction}

All the production equipment on the CTM production line is the key equipment. If the equipment operation fault occurs, the entire production line will be terminated. So monitoring and reading the real-time status of the production equipment is important. In order to realize the automation of CTM production, the equipment must be able to accept the production order. In order to achieve these requirements, the equipment must be able to exchange data with the upper application.

There are three principal methods of interaction with the production equipment: Driver, Dynamic Data Exchange (DDE), OLE for Process Control (OPC). The characteristic of the three methods will be introduced respectively as followed.

1) Driver: The method writes a different driver for unusual equipment in order to realize the interaction with the production equipment, which has the characteristics of fast communication, high flexibility, high customization, easy to implement. However, the drawback of this method is also obvious. It will take a lot of time to write a variety of drivers for a lot of equipment. When the hardware upgrade, the software system has to change to adapt to the hardware, software and hardware coupling is too high. Due to different upper application write a separate driver for equipment. Different upper application can't access equipment at the same time. It will cause the equipment access conflicts.

2) $D D E$ : DDE realizes the data exchanges between programs that developed based on the Microsoft Win32 
application programming interface (API) through shared memory. It can update the data between the applications automatically [3]. Hardware manufacturers develop the corresponding application for their equipment. The application can provide service for upper application through DDE. This method solves the problem of the Driver that has to develop diverse driver different equipment. Nevertheless, binding equipment and DDE procedures make the equipment buyer have to consider the availability of the application on the equipment when they choose the equipment. DDE data exchange based on message mechanism of Windows uses the Client/Server model. This method has advantages of reliable real-time performance, easy to implement the connection with control network and information network. Nonetheless, because of DDE is based on message mechanism of Windows, which requires the control network and information network have the function of Windows DDE. When the traffic is enormous, the shared memory communication mechanism of DDE will cause a slow refresh, crash, etc. The mechanism based on memory lacks the guarantee of safety, and reliability is low.

3) OPC: OPC is based on Microsoft's COM/DCOM technology and DNA (Distributed Internet Application) architecture. It offers a standard data communication mechanism between the management application and the automation control system or the equipment [4]. OPC uses the Client/Server model. It establishes a set of rules between the equipment manufacturer and the software developer, rather than specific implementation. The implementation code depends on the method of the upper application access the equipment and the data processing. Following the OPC standard, the software developer can eliminate the differences of separate equipment. The upper application can get the data through the OPC service interface provided by the equipment. At the same time, the equipment manufacturers do not need to consider the discrete software requirement and communication protocol [5]. Compare to the Driver, OPC completely shields the differences of the underlying hardware equipment. Relative to the DDE, OPC encapsulates the equipment data into unified OPC format. The formatted data are provided to the upper application through service. In this way, large amounts of data are exchanged between the upper application and the equipment. Because based on COM and DCOM, the OPC technology has a lot of advantages, such as high data transfer performance, good safety management performance, low development cost, etc.

There are a lot of different equipment will participate in the CTM production process. The upper application needs to read a lot of data from the equipment. The Driver and the DDE can't meet these requirements. The OPC supports large amounts of data interaction, and development effort is small, so the system will take OPC as the data interactive method between upper application and equipment.

As the OPC DA interface provided by the traditional OPC technology is based on the Microsoft COM/DCOM technology, so the OPC is difficult to provide services to the different operating systems on the Internet platform. At the same time, due to DCOM does not use port 80 , while HTTP communication is conducted through port 80 , which hinders the upper applications access the services provided by OPC through the Internet[6].

In order to solve this problem, the OPC foundation proposed OPC XML-DA technology. OPC XML-DA is based on the XML specification and SOAP technology. The data that want to be transferred through the Internet will be organized as the SOAP message. As data format on the Ethernet is OPC DA and on the Internet is OPC XML-DA, those two data formats can't automatic transfer. In order to solve the problem, the data intermediate software is necessary. Through the data intermediate software, the equipment can provide OPC DA data to upper application and the upper application can convey the instructions to equipment use OPC XML-DA data as well. Thereby the upper application on the Internet can read and control the equipment.

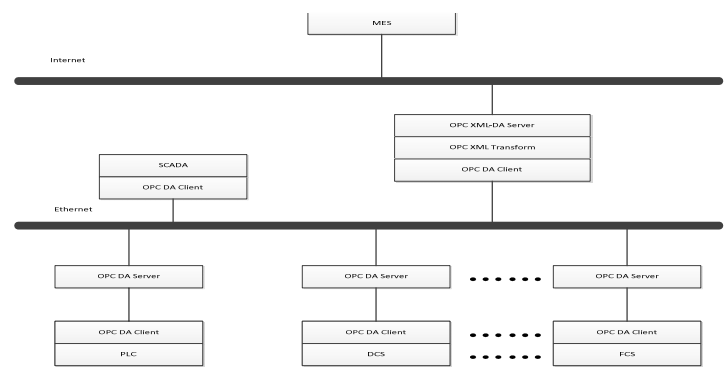

Figure 1. Information interaction diagram

\section{B. SCADA}

The production process of CTM must have a strict quality control, which requires the production operation in the production process must follow the production specifications. Each link of the production process is under effective control. If all the work is done by manpower, not only it will bring huge workload for workshop staff, but also the results of observation are not accurate enough, all the equipment can't be monitored at all time in the whole manufacturing process. In order to monitor all the equipment in the entire CTM production process, an automatic monitoring system is necessary.

Nowadays, there are three automatic monitoring systems are wildly used. They are PLC, DCS, SCADA.

PLC (Programmable Logic Controller) is widely used as the industrial field measurement control. It has many benefits, such as strong field measurement and control function, stable performance, high reliability, mature technology, but PLC focuses on the monitoring of a single device, lacks the capacity of comprehensive coordination between equipment.

DCS (Distributed Control System) is suitable for the industrial field that has many measurements and control points, needs high control precision and fast measurement. The characteristic of DCS is decentralized control and centralized monitoring. Compare to PLC, DCS can make an overall consideration when DCS controls the equipment. DCS has the ability to interconnect equipment through the network, and is easy to extend, has superior security features. 
Nevertheless, the field data acquisition and control unit of the DCS are usually located in a restricted area. The unit of DCS communicates with each other through a reliable and high-speed network (LAN). It is difficult to integrate with other Control System through the Internet.

SCADA (Supervisory Control And Data Acquisition) can realize Data Acquisition, equipment control, measure, parameters adjustment, all kinds of signal alarm and other functions. SCADA system is focused on monitoring and control. Compared with DCS, SCADA can integrate a variety of measurement and control products of different manufacturers, supports for multiple communication protocols, so the SCADA System has better openness. Equipment that controlled by SCADA can be distributed to multiple locations. Usually the SCADA System is used at the top management level of monitoring data. In the production process of CTM, the automatic monitoring system needs to integrate with upper application (such as MES) through Internet. A variety of plants and equipment needs to be managed by an automated monitoring system. If use PLC or DCS, it will be difficult to integrate with upper application and make a multi-plant, multi-device cooperative scheduling. In summary, the system will take SCADA as the CTM production automatic monitoring system.

The literature [7] puts forward a kind of typical SCADA hardware architecture and software architecture, and makes a detail introduction of the function of each part. On the basis of the literature, this paper designs the modules of the SCADA. As CTM production process has the requirements that monitoring the real-time production status, analyzing the real-time production data and fault alarm, the SCADA has the real-time monitoring module, process analysis module, alarm module. Those modules are based on a real-time database. Due to the historical production process of CTM must be able to backtrack and the historical data must be able to query and used to analyze in order to optimize the production process, the history database module is designed for the SCADA.

The SCADA consists of three layers, from bottom to top, is collecting layer, service layer, control layer.

1) Collecting layer: The collecting layer will collect the data of the equipment, such as environmental control equipment, inspection equipment, weighing equipment. The equipment will collect the data that the real-time database needs. The collected data will be stored in the equipment acquisition station. The equipment acquisition station will transmit the data to the data acquisition module in the control layer periodically through an OPC interface. In the end, the data will be transmitted to the real-time database.

2) Control layer: Control layer is the core of the SCADA system. It consists of the process monitoring module, data analysis module, fault alarm module, history database management module, and other modules. The principal job of the control layer is to real-time monitor the production process, display data trends, analyze the process data, adjusting the process parameters. The control layer also has to monitor the equipment operating status. If the equipment operating status is abnormal, the fault alarm has to issue a warning equipment failure. All the history will be stored in the history database. The core of the control layer is the real-time database. It provides real-time data support to other modules.

3) Service layer: Service layer implements the SCADA system and the upper application data interactive interface. The interface gets the data from the real-time database and the history database, and then, provides data service to the upper application. The type of communication is publishsubscribe. The SCADA system sends the encapsulated data to the data subscription application by means of the broadcast or multicast. This approach is beneficial to improve the efficiency of network communication, and have high real-time performance, meet the real-time requirements for the upper application.

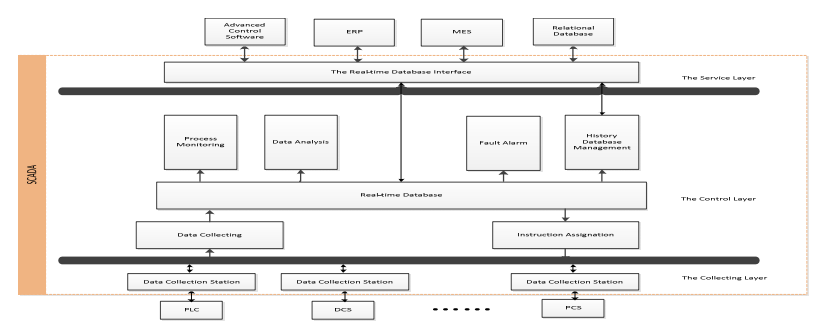

Figure 2. SCADA system architecture figure

\section{MES}

In the management of CTM production process, there are several problem is often appeared.

- Workshop dispatchers don't have all the information of the available resource, lead to unreasonable resource scheduling.

- Workshop production scheduling plan isn't scientific, lead to productive resource or underutilization, or contention.

- The person in charge of every work stage in the CTM production process can't get the status of previous work stage, lead to information isolated island between work stages.

- The material isn't fully prepared before production. Material review procedure isn't comprehensive. material flow in the production isn't clear.

- Production data record is inaccurate.

- Record data is not properly kept.

- The data that spend a lot of manpower, material resource collected is not fully utilized.

These problems are caused by poor production management. The solution of these problems is to set up a production management system.

Nowadays, there are a lot of production management ideas, for example, ERP, JIT, OPT, and so on. However, most of the production management ideas are focused on the management of the production plan and the plan implementation, and the workshop production control ability is very weak. How to improve the adaptability of production 
planning, increase the flow of the production process information and improve the real-time and flexibility of the implementation of the production plan, has become an important research subject.

In order to solve those problems, MESA (Manufacturing Execution System Association International) put forward the concept of MES (Manufacturing Execution Systems) in the $1990 \mathrm{~s}$.

MESA of MES is defined as follows: MES deliver information that enables the optimization of production activities from order launch to finished goods. Using current and accurate data, MES guides, initiates, responds to, and reports on plant activities as they occur. The resulting rapid response to changing conditions, coupled with a focus on reducing non value-added activities, drives effective plant operations and processes. MES improves the return on operational assets as well as on-time delivery, inventory turns, gross margin, and cash flow performance. MES provides mission-critical information about production activities across the enterprise and supply chain via bidirectional communications [8].

MES is the bridge of Enterprise Resource Planning (ERP) and Process Control System (PCS). According to the production plan made by ERP and the existing production resource, MES can make reasonable production arrangement. According to the feedback of the production, MES can get and analyze the production data, and then make a real-time production scheduling.

MESA proposed MES functional components and integration model in 1997 [9]. According to the traditional structure of the MES, the production process of CTM and considering the quality requirements of CTM production process, this paper design the module of MES.

1) Master data management: Master data management includes organization management, personnel management, container management, equipment management, material management. Master data is the basis of the normal operation of the system. In the system operating, master data must be present and correct maintenance.

2) System management: System management includes account management, authority management, remind management, system log management. Accounting management includes account application, account update. Authority management includes set different permissions level, grant appropriate permission to different account. Remind management includes set the time of the reminder, set the people the needs to remind, set different remind ways such as SMS (Short Messaging Services) reminder, email reminder, etc. System log management includes personnel system operating record, history operation records query.

3) Plan management: Plan management includes yearly production plan management, monthly production plan management, workshop production plan management, workshop scheduling. All the submodules of plan management have the function of add plan and update plan. The workshop production plan is made refer to monthly production plan, and the monthly production plan refers to yearly production plan. The function of workshop scheduling is to make a systematic scheduling for all batch according to the workshop production plan. Currently, there are a variety of scheduling methods, such as method based on operational research, genetic algorithm, method based on heuristic rules, simulation method, method based on DEDS, method based on artificial intelligence, etc[10]. As the genetic algorithm has superior global search ability, and is easy to implement, so the genetic algorithm will be used to solve the workshop batch scheduling problem. Combined with the characteristic of CTM production process that has many work stages in one batch production process, the genetic algorithm will use an encoding style based on work stage[13]. The evaluation method of the genetic algorithm is the batch production time of each possible solution.

4) Workshop management: Workshop management includes process route management, prescription management, batch information management, work stage management. Because different CTM has different process route and the CTM production process must follow the process route, the module of process route management will create and configure process route for different CTM production process and maintain the process route. The function of prescription management is to make prescription to record the required materials kinds and the material quantity according to the different batch production requirements. The prescription management module will maintain the prescription. Batch information management is to create batch instruction for different batchs. The batch instruction records the current execution status of different batchs. Batch information management will update the batch instruction. The batch production process will be tracked through the batch instruction. The batch production process is consists of some work stage. The work stage management module will give a work stage instruction according to the process route and the current production status recorded by batch instruction to the workshop and collect the work stage process data and the result.

5) Interface management:Interface management includes equipment interface management, the third party system interface management. MES interacts with the equipment through the equipment interface. In order to interacts with other system,such as raw package management system, warehousing system, document generation system, the third party system interface management module will generate and maintain the interface with other system.

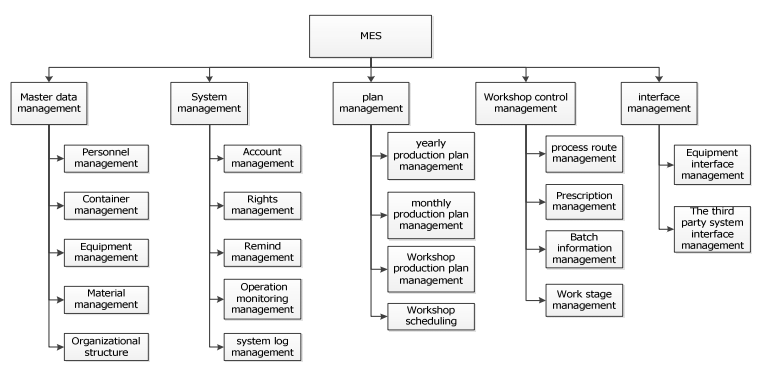

Figure 3. MES function block diagram 


\section{SYSTEM INTEGRATION}

The Figure 4 depicts the architecture of the System. The MES work out the production plan according to the technological procedure and process route of the CTM. The production instruction will be given to the SCADA System from the MES through OPC. The SCADA System monitors the equipment according to the production instruction. The SCADA System will collect the equipment data through the OPC and provide the data to the MES. The MES will analyze the data and determine the current state of the CTM production process. The MES will give the resulting production instruction combining with the current production status and the process route.

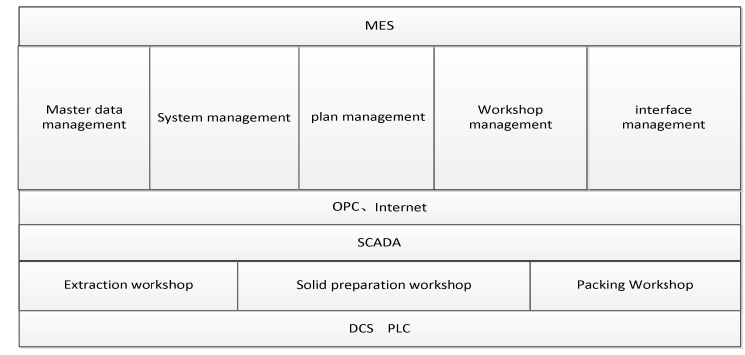

Figure 4. System architecture diagram

\section{CONCLUSION}

In view of the CTM production has many problems such as low production automation, data collection difficulty, disorder of the workshop production scheduling, this paper proposes integrating SCADA system and MES system through OPC technology to establish the CTM production management system architecture. Future jobs include: (1) establish the process optimization module, use statistics to analyze the historical production data and find out the process route of nodes that can be optimized. (2) improve the performance of scheduling algorithm.

\section{ACKNOWLEDGMENT}

We thank Software Engineering Institute in BeiHang University for their contribution to the paper.

\section{REFERENCES}

[1] XIONG Mao-hua,YU Hong-qing.Research on MIS-based Interlligent ERP for Large \& Middle Sized Manufacturing Medicine Enterprise[J].JI SUAN JI YU XIAN DAI HUA.2006(8):84-86J.

[2] GAO Xue-jin,Wang Pu,ZHANG Hui-qing,et al. Study of MES for Pharmacy Industry [J].AUTOMATION PANORAMA.2005.22(6)

[3] Jiang Wei.Implementing Data Exchange between WinCC and Excel with DDE Technology.NAN GANS TECHNOLOGY AND MANAGEMENT.2011(1):56

[4] ZHANG Jun,ZOU Yi-ren,MA Zeng-liang.Design and Implement of OPC XML-DA Gateway [J].APPLICATION RESEARCH OF COMPUTERS,2005,22(7)

[5] CAI Xiang-yun,ZHENG Xiao-hu,JIANG Lin.THE Development and Application of OPC Specification[J].Journal of Kunming University of Science and Technology,2002.6.

[6] ZHU Xiao-mei,HAN Hong-jun.Research On Industrial Control System Integration Based On XML And OPC [J].CHINA SCIENCE AND TECHNOLOGY INFORMATION.2006(2):11-14.

[7] Daneels.A, CERN, Geneva,et al. WHAT IS SCADA?.[J]. International Conference on Accelerator and Large Experimental Physics Control Systems,1999

[8] MESA International. MES Explained: A High Level Vision [R]. Pittsburgh, USA: MESA International White Paper Number6, 1997.

[9] MESA International.MES Functionalities \& MRP to MES Data Flow Possibllities.1997.

[10] MA Zheng-yuan,WANG Wei-ling,WANG Yu-sheng.Systematic Research and Development On the Production Scheduling [J]. GROUP TECHNOLOGY \& PRODUCTION MODERNIZATION. 2005(22):10-14. 